This item was submitted to Loughborough's Research Repository by the author.

Items in Figshare are protected by copyright, with all rights reserved, unless otherwise indicated.

\title{
Coherence scanning interferometry: linear theory of surface measurement
}

PLEASE CITE THE PUBLISHED VERSION

http://dx.doi.org/10.1364/AO.52.003662

\section{PUBLISHER}

(c) Optical Society of America

\section{VERSION}

VoR (Version of Record)

\section{LICENCE}

CC BY-NC-ND 4.0

\section{REPOSITORY RECORD}

Coupland, Jeremy M., Rahul Mandal, Kanik Palodhi, and Richard K. Leach. 2019. "Coherence Scanning Interferometry: Linear Theory of Surface Measurement”. figshare. https://hdl.handle.net/2134/13310. 


\title{
Coherence scanning interferometry: linear theory of surface measurement
}

\author{
Jeremy Coupland, ${ }^{1, *}$ Rahul Mandal, ${ }^{1}$ Kanik Palodhi, ${ }^{1}$ and Richard Leach ${ }^{2}$ \\ ${ }^{1}$ Mechanical and Manufacturing Engineering, Loughborough University, Loughborough, LE11 3TU, UK \\ ${ }^{2}$ Engineering Measurement Division, National Physical Laboratory, Teddington, TW11 OLW, UK \\ ${ }^{*}$ Corresponding author: j.m.coupland @ lboro.ac.uk
}

Received 21 December 2012; revised 4 April 2013; accepted 15 April 2013;

posted 23 April 2013 (Doc. ID 182295); published 22 May 2013

\begin{abstract}
The characterization of imaging methods as three-dimensional (3D) linear filtering operations provides a useful way to compare the 3D performance of optical surface topography measuring instruments, such as coherence scanning interferometry, confocal and structured light microscopy. In this way, the imaging system is defined in terms of the point spread function in the space domain or equivalently by the transfer function in the spatial frequency domain. The derivation of these characteristics usually involves making the Born approximation, which is strictly only applicable to weakly scattering objects; however, for the case of surface scattering, the system is linear if multiple scattering is assumed to be negligible and the Kirchhoff approximation is assumed. A difference between the filter characteristics derived in each case is found. However this paper discusses these differences and explains the equivalence of the two approaches when applied to a weakly scattering object. (c) 2013 Optical Society of America

OCIS codes: (070.0070) Fourier optics and signal processing; (090.0090) Holography; (120.0120) Instrumentation, measurement, and metrology; (180.0180) Microscopy; (240.0240) Optics at surfaces; (290.0290) Scattering.

http://dx.doi.org/10.1364/AO.52.003662
\end{abstract}

\section{Introduction}

Coherence scanning interferometry (CSI) is a threedimensional (3D) imaging technique that is used to measure areal surface topography. It combines the vertical resolution of an interferometer with the lateral resolution of a high-power microscope and provides a fast, noncontacting alternative to contact stylus profilometers [1-3]. CSI typically utilizes broadband, incandescent, or LED sources and Mirau interference objectives to record the interference between the light scattered by the object and that reflected from a reference surface as the objective is scanned though focus [4]. Since the source illumination is limited in both temporal and spatial coherence, the interference fringes are observed over a finite scan range and it is relatively straightforward

$1559-128 X / 13 / 163662-09 \$ 15.00 / 0$

(C) 2013 Optical Society of America to locate the bright zero-order fringe that identifies when path length is balanced in the interferogram. For this reason, CSI is particularly useful for the measurement of discontinuous surfaces, such as those produced in the microelectronics industry.

Despite these significant advantages, CSI exhibits certain problems that restrict its use as a traceable measurement tool particularly when it is used to measure sloped artifacts [5]. Since CSI instruments are typically calibrated using step height standards and lateral calibration artifacts of a "waffle plate" design [6] , problems with the measurement of sloped artifacts often go unnoticed $[\underline{7,}, \underline{8}]$. In order to overcome these deficiencies and improve measurement quality a calibration and adjustment method using spherical artifacts has recently been considered [9-11]. Since all slope angles are equally represented by a spherical surface (smaller than the field of view of the CSI), slope-related errors are immediately apparent. More importantly, however, an interferogram 
of a spherical artifact provides the information necessary to define the resolution of the instrument and, in some cases, compensate for errors introduced by lens aberrations [9].

The theory that underpins this work is based on a linear theory of 3D imaging that was first published in the context of optical holography by Wolf [12], and Dandliker and Weiss [13]. Using this approach the performance of 3D imaging techniques including digital holography, confocal microscopy, CSI, and other interferometers can be compared in terms of linear system theory [14-18]. In this way the system is characterized either in the space domain by the point spread function (PSF) or equivalently in the (spatial) frequency domain, by the transfer function (TF). As the image of a point-like object, the PSF provides a direct measure of the 3D "resolution cell" of the imaging system while the $\mathrm{TF}$ describes how the phase and amplitude of the spatial frequencies present within the object are modified by the imaging system. For the case of CSI, the phase of the TF is of primary importance since the surface height is deduced from this quantity.

Although the linear theory provides a good means to compare the theoretical performance of different imaging systems, it rests on the assumption of weak scattering and the validity of the Born approximation [19]. In essence, weak scattering implies that the object causes a small perturbation to the illuminating field. The Born approximation can be assumed when there are small changes in refractive index or small objects, such as particles suspended in fluid, but is not generally applicable to the comparatively large changes in refractive index that are typical of $3 \mathrm{D}$ scattering objects. When light is scattered from the interface between homogenous media, however, it is not necessary to assume the Born approximation and, providing that there is no multiple scattering and the surface is smooth at the optical scale, the process is also linear. A detailed analysis of surface scattering has been presented by Beckmann and Spizzichino [20] and this forms the basis of inverse scattering methods that attempt to deduce surface topography from measurements of the scattered field [21-23]. In this case, the surface boundary conditions are assumed and the object can be replaced by an infinitely thin foil-like object, which follows the surface topography and henceforth will be called the "foil model" of the surface. As pointed out by Sheppard in the context of confocal microscopy $[24,25]$, an apparent consequence of the surface scattering approach is that the effective TF (and PSF) of the measuring instrument is modified.

In this paper, the derivation of the foil model of the surface is presented and the associated PSF and TF are defined. Starting from the integral form of the Helmholtz equation, the differences between the analyses based on the Born approximation and the surface scattering approach are contrasted. It is shown that there is a small but significant difference between the PSFs and TFs; however, the two approaches yield exactly the same result when both the numerical aperture and refractive change tend to zero.

\section{Theory}

It is shown elsewhere [17] that the output, $O_{B}(\mathbf{r})$, of a CSI instrument can be written as a 3D linear filtering operation that is characterized in the space domain by the convolution,

$$
O_{B}(\mathbf{r})=\int H_{B}\left(\mathbf{r}-\mathbf{r}^{\prime}\right) \Delta_{B}\left(\mathbf{r}^{\prime}\right) \mathrm{d}^{3} r^{\prime},
$$

where $\Delta_{B}(\mathbf{r})=4 \pi^{2}\left(1-n^{2}(\mathbf{r})\right)$ defines the object in terms of the refractive index $n$ and $H_{B}\left(\mathbf{r}-\mathbf{r}^{\prime}\right)$ is the PSF given by

$$
H_{B}(\mathbf{r})=\int G_{\mathrm{NA}}^{2}\left(\mathbf{r}, k_{0}\right) k_{0}^{2} S\left(k_{0}\right) \mathrm{d} k_{0} .
$$

In this expression, $S\left(k_{0}\right)$ is the spectral density expressed as a function of the wavenumber $k_{0}$ and $G_{\mathrm{NA}}\left(\mathbf{r}, k_{0}\right)$ is the PSF of an imaging system of numerical aperture $N_{A}$ given by

$$
\begin{aligned}
G_{\mathrm{NA}}\left(\mathbf{r}, k_{0}\right)= & \int \frac{j}{4 \pi k_{0}} \delta\left(|\mathbf{k}|-k_{0}\right) \text { step } \\
& \times\left(\frac{\mathbf{k} \cdot \hat{\boldsymbol{o}}}{k_{0}}-\sqrt{1-N_{A}^{2}}\right) e^{j 2 \pi \mathbf{k} \cdot \mathbf{r}} \mathrm{d}^{3} k,
\end{aligned}
$$

where $\hat{o}$ is a unit vector in the direction of the optical axis and $\delta(x)$ and $\operatorname{step}(x)$ represent a Dirac delta function and a Heaviside step function, respectively. Equivalently the filtering operation is defined in the frequency domain ( $k$-space) by the relation

$$
\tilde{O}_{B}(\mathbf{k})=\tilde{\Delta}_{B}(\mathbf{k}) \tilde{H}_{B}(\mathbf{k}),
$$

where tilde denotes Fourier transformation such that $\tilde{H}_{B}(\mathbf{k})=\int H_{B}(\mathbf{r}) e^{-j \pi 2 \mathbf{k} \cdot \mathbf{r}} \mathrm{d}^{3} k$ represents the $\mathrm{TF}$ and is given by

$$
\tilde{H}_{B}(\mathbf{k})=\iint \tilde{G}_{\mathrm{NA}}\left(\mathbf{k}^{\prime}, k_{0}\right) \tilde{G}_{\mathrm{NA}}\left(\mathbf{k}-\mathbf{k}^{\prime}, k_{0}\right) \mathrm{d}^{3} k^{\prime} k_{0}^{2} S\left(k_{0}\right) \mathrm{d} k_{0},
$$

where

$$
\tilde{G}_{\mathrm{NA}}\left(\mathbf{k}, k_{0}\right)=\frac{j}{4 \pi k_{0}} \delta\left(|\mathbf{k}|-k_{0}\right) \operatorname{step}\left(\frac{\mathbf{k} \cdot \hat{\boldsymbol{o}}}{k_{0}}-\sqrt{1-N_{A}^{2}}\right) .
$$

It is noted that Eqs. (1)-(6) differ slightly from those given in reference [17], as some numerical constants have been included in the object function and the alternative definition of wavenumber $k_{0}=$ $1 / \lambda$ has been used here. The equations rest on the 
assumption of weak scattering, or in other words, that the incident field is weakly perturbed by the object. This is reasonable for objects that are characterized by small variations in refractive index, such as cellular tissue, but is rarely justified for general $3 \mathrm{D}$ objects. For the case of strong surface scattering from the interface between two homogenous media however, providing multiple scattering is negligible, the process can also be considered linear. In order to relate these two apparently disparate processes scattering by an object characterized by the function $\Delta_{B}(\mathbf{r})=4 \pi^{2}\left(1-n^{2}(\mathbf{r})\right)$ is considered, as shown in Fig. 1.

If the object is illuminated by the reference field $E_{r}(\mathbf{r})$, then scattered field denoted by $E_{s}(\mathbf{r})$, is given by the integral form of the Helmholtz equation such that [17]

$$
E_{s}(\mathbf{r})=k_{0}^{2} \int G\left(\mathbf{r}-\mathbf{r}^{\prime}\right) \Delta_{B}\left(\mathbf{r}^{\prime}\right)\left(E_{s}\left(\mathbf{r}^{\prime}\right)+E_{r}\left(\mathbf{r}^{\prime}\right)\right) \mathrm{d}^{3} r^{\prime},
$$

where $G(\mathbf{r})=e^{j 2 \pi k|\mathbf{r}|} / 4 \pi|\mathbf{r}| ;$ is the free-space Green's function that defines a point source. It is noted that the scattered field is in general a nonlinear function of the object function $\Delta_{B}\left(\mathbf{r}^{\prime}\right)$; however, the process is linearized by assuming that the term $E_{s}\left(\mathbf{r}^{\prime}\right)$; in the integrand is negligible. This is the well-known Born approximation, which is applicable to weak scattering events [19]. It is clear, however, that the only contribution to the integral is from regions where $\Delta_{B}\left(\mathbf{r}^{\prime}\right)=4 \pi^{2}\left(1-n^{2}\right)$ is nonzero (i.e., from the volume occupied by the object itself) and the scattered field can, therefore, be written as the volume integral,

$$
E_{s}(\mathbf{r})=k_{0}^{2} \int_{V} G\left(\mathbf{r}-\mathbf{r}^{\prime}\right) \Delta_{B}\left(\mathbf{r}^{\prime}\right) E_{t}\left(\mathbf{r}^{\prime}\right) \mathrm{d}^{3} r^{\prime}
$$

where $E_{t}(\mathbf{r})$ is the transmitted field (i.e., that inside the object boundary) and $V$ denotes the object volume.

Since inside the object $\left(\nabla^{2}+4 \pi^{2} n^{2} k_{0}^{2}\right) E_{t}\left(\mathbf{r}^{\prime}\right)=0$ and $\left(\nabla^{2}+4 \pi^{2} k_{0}^{2}\right) G\left(\mathbf{r}-\mathbf{r}^{\prime}\right)=0$, then $G\left(\mathbf{r}-\mathbf{r}^{\prime}\right) E_{t}\left(\mathbf{r}^{\prime}\right)=$ $\left(1 / 4 \pi^{2} k_{0}^{2}\left(n^{2}-1\right)\right)\left(E_{t}\left(\mathbf{r}^{\prime}\right) \nabla^{2} G\left(\mathbf{r}-\mathbf{r}^{\prime}\right)-G\left(\mathbf{r}-\mathbf{r}^{\prime}\right) \nabla^{2} E_{t}\left(\mathbf{r}^{\prime}\right)\right)$.

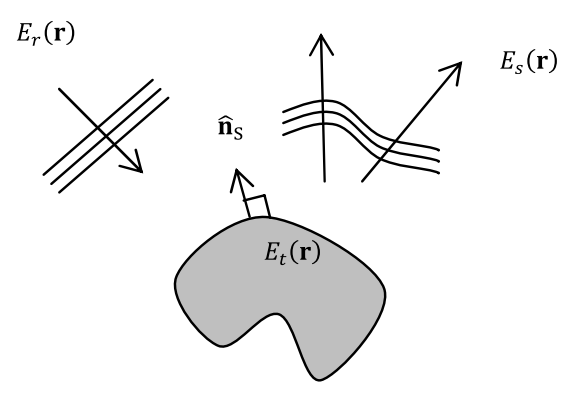

Object $\Delta_{B}(\mathbf{r})$

Fig. 1. Scattering from a $3 \mathrm{D}$ object.
Substitution gives

$$
E_{s}(\mathbf{r})=\int_{V}\left(G\left(\mathbf{r}-\mathbf{r}^{\prime}\right) \nabla^{2} E_{t}\left(\mathbf{r}^{\prime}\right)-E_{t}\left(\mathbf{r}^{\prime}\right) \nabla^{2} G\left(\mathbf{r}-\mathbf{r}^{\prime}\right)\right) \mathrm{d}^{3} r^{\prime}
$$

and applying Green's theorem it is found

$$
E_{s}(\mathbf{r})=\int_{S}\left(G\left(\mathbf{r}-\mathbf{r}^{\prime}\right) \frac{\partial E_{t}\left(\mathbf{r}^{\prime}\right)}{\partial n}-E_{t}\left(\mathbf{r}^{\prime}\right) \frac{\partial G\left(\mathbf{r}-\mathbf{r}^{\prime}\right)}{\partial n}\right) \mathrm{d} s,
$$

where $S$ denotes the object boundary. Equation (10) is the Kirchhoff integral [19]. It is exact, but hardly surprising, as it merely shows the well-known result that the scattered field from the medium can be written purely in terms of the field at the object boundary. However, it is now straightforward to linearize the scattering process by assuming appropriate boundary conditions. Following Beckman and Spizzichino [20], if the surface is illuminated by a unit amplitude plane wave, propagating with wave vector, $\mathbf{k}_{r}$, such that $E_{r}(\mathbf{r})=e^{2 \pi j \mathbf{k}_{\mathbf{r}} \cdot \mathbf{r}}$, the boundary field and its normal derivative can be written [20],

$$
\begin{gathered}
E_{t}(\mathbf{r})=(1+R) e^{2 \pi j \mathbf{k}_{\mathbf{r}} \cdot \mathbf{r}}, \\
\frac{\partial E_{t}(\mathbf{r})}{\partial n}=2 \pi j \mathbf{k}_{\mathbf{r}} \cdot \hat{\mathbf{n}}_{S}(1-R) e^{2 \pi j \mathbf{k}_{\mathbf{r}} \cdot \mathbf{r}},
\end{gathered}
$$

where $\hat{\mathbf{n}}_{S}$ is the outward surface normal (as shown in Fig. 1 ) and $R$ is the Fresnel amplitude reflection coefficient, which is assumed to be constant over the range of scattering angles of interest. Beckmann and Spizzichino have discussed the validity of these boundary conditions in detail [20] but for the purposes of this paper it is noted that:

(i) The surface must be slowly varying on the optical scale such that the local radius of curvature is more than the wavelength. This is the Kirchhoff or physical optics approximation [19].

(ii) For a perfect conductor the reflection coefficient is indeed constant $(R=1)$.

(iii) More generally, the reflection coefficient depends on polarization but the sum of reflection coefficients for orthogonal polarization states is approximately constant for angles of incidence that are less than $45 \mathrm{deg}$.

(iv) For a dielectric, the field at the lower boundary and its gradient may depart markedly from those given in Eqs. (11) and (12) due to propagation through the object. However, this component of the field will generally be separable from that scattered from the top boundary using CSI due to the extra path length traveled.

In order to explain the output of a CSI instrument it is first necessary to consider the process of far-field imaging - that is, measuring or reconstructing a field 
solely from the information present at a distant boundary. It is shown in Appendix A that propagation to and from a distant boundary is a linear filtering operation that is characterized by a PSF that depends on the numerical aperture of the instrument. In the following a similar process will be followed to give an expression for the measured scattered field.

First, consider the field that propagates from the upper surface to a point $\mathbf{r}_{\mathbf{b}}$; on a distant boundary $\Sigma$, as shown in Fig. 2. Since the boundary is at a large distance, $\mathbf{r}_{\mathbf{b}} \gg \mathbf{r}$ and the far-field Green's function can be written

$$
G\left(\mathbf{r}_{\mathbf{b}}-\mathbf{r}\right) \approx \frac{e^{2 \pi j k_{0}\left|\mathbf{r}_{\mathbf{b}}\right|}}{4 \pi\left|\mathbf{r}_{\mathbf{b}}\right|} e^{-2 \pi j k_{0} \mathbf{r} \cdot \frac{\mathbf{r}_{\mathbf{b}}}{\mathbf{r}_{\mathbf{b}}}}
$$

The normal derivative of the Green's function is, therefore,

$$
\frac{\partial G\left(\mathbf{r}_{\mathbf{b}}-\mathbf{r}\right)}{\partial n}=-2 \pi j G\left(\mathbf{r}_{\mathbf{b}}-\mathbf{r}\right) k_{0} \frac{\mathbf{r}_{\mathbf{b}}}{\left|\mathbf{r}_{\mathbf{b}}\right|} \cdot \hat{\mathbf{n}}_{S} .
$$

Substituting Eqs. (11)-(14) into the Kirchhoff integral of Eq. (10), the scattered field at the distant boundary is given by

$$
\begin{aligned}
E_{s}\left(\mathbf{r}_{\mathbf{b}}\right)= & j \frac{e^{2 \pi j k_{0}\left|\mathbf{r}_{\mathbf{b}}\right|}}{2\left|\mathbf{r}_{\mathbf{b}}\right|} \int_{S} e^{-2 \pi j\left(k_{0} \cdot \frac{\mathbf{r}_{\mathbf{b}}}{\left|\mathbf{r}_{\mathbf{b}}\right|} \mathbf{k}_{\mathbf{r}} \cdot \mathbf{r}\right)}\left[R\left(k_{0} \frac{\mathbf{r}_{\mathbf{b}}}{\left|\mathbf{r}_{\mathbf{b}}\right|}-\mathbf{k}_{\mathbf{r}}\right)\right. \\
& \left.+\left(k_{0} \frac{\mathbf{r}_{\mathbf{b}}}{\left|\mathbf{r}_{\mathbf{b}}\right|}+\mathbf{k}_{\mathbf{r}}\right)\right] \cdot \hat{\mathbf{n}}_{S} \mathrm{~d} s .
\end{aligned}
$$

In accordance with comment (iv) above, a region of interest on the upper surface of the object can be defined by the function, $A(\mathbf{r})$, given by

$$
A(\mathbf{r})=W\left(r_{x}, r_{y}\right) \delta\left(r_{z}-s\left(r_{x}, r_{y}\right)\right),
$$

where $W\left(r_{x}, r_{y}\right)$ is a window function. Using the sifting properties of the Dirac delta function [26], the

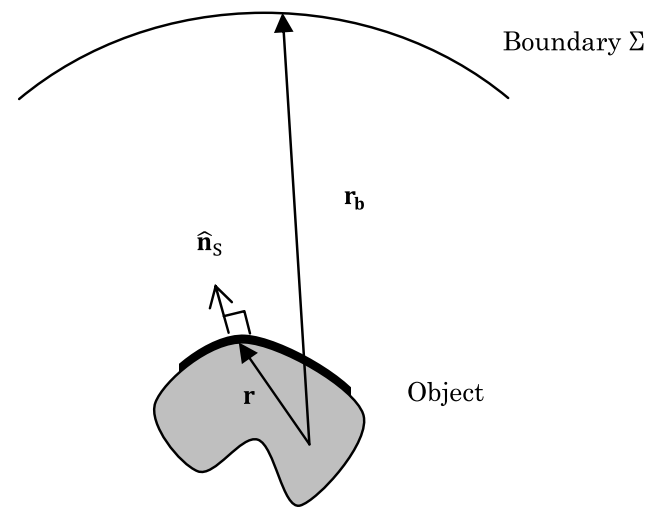

Fig. 2. Surface scattering to a distant boundary. scattered field can be written as an indefinite integral such that,

$$
\begin{aligned}
E_{s}\left(\mathbf{r}_{\mathbf{b}}\right)= & j \frac{e^{2 \pi j k_{0}\left|\mathbf{r}_{\mathbf{b}}\right|}}{2\left|\mathbf{r}_{\mathbf{b}}\right|} \int e^{-2 \pi j\left(k_{0} \frac{\mathbf{r}_{\mathbf{b}}}{r_{\mathbf{b}} \mid} \mathbf{k}_{\mathbf{r}}\right) \cdot \mathbf{r}}\left[R\left(k_{0} \frac{\mathbf{r}_{\mathbf{b}}}{\left|\mathbf{r}_{\mathbf{b}}\right|}-\mathbf{k}_{\mathbf{r}}\right)\right. \\
& \left.+\left(k_{0} \frac{\mathbf{r}_{\mathbf{b}}}{\left|\mathbf{r}_{\mathbf{b}}\right|}+k_{r}\right)\right] \cdot \hat{\mathbf{n}}_{S} \frac{A(\mathbf{r})}{\hat{\mathbf{n}}_{S} \cdot \mathbf{z}} \mathrm{d}^{3} r .
\end{aligned}
$$

If it is assumed that this field can be measured, for example using digital holography, an expression for the measured field can be obtained. In a similar manner to the derivation presented in Appendix A, the measured field, $E_{m}^{\prime}\left(\mathbf{r}^{\prime}\right)$, can be written as the Kirchhoff integral,

$E_{m}^{\prime}\left(\mathbf{r}^{\prime}\right)=\int_{\Sigma}\left[G^{*}\left(\mathbf{r}^{\prime}-\mathbf{r}_{\mathbf{b}}\right) \frac{\partial E_{s}\left(\mathbf{r}_{\mathbf{b}}\right)}{\partial n}-E_{s}\left(\mathbf{r}_{\mathbf{b}}\right) \frac{\partial G^{*}\left(\mathbf{r}^{\prime}-\mathbf{r}_{\mathbf{b}}\right)}{\partial n}\right] \mathrm{d} s$.

Using the far-field Green's function and assuming, without loss of generality, that the boundary surface is spherical,

$$
\begin{aligned}
E_{m}^{\prime}\left(\mathbf{r}^{\prime}\right)= & \frac{-k_{0}}{2} \int_{\Sigma} \frac{1}{\left|\mathbf{r}_{\mathbf{b}}\right|^{2}} \int e^{-2 \pi j\left(k_{0} \frac{\mathbf{r}_{\mathbf{b}}}{\mid \mathbf{r}_{\mathbf{b}}} \mid \mathbf{k}_{\mathbf{r}}\right) \cdot \mathbf{r}}\left[R\left(k_{0} \frac{\mathbf{r}_{\mathbf{b}}}{\left|\mathbf{r}_{\mathbf{b}}\right|}-\mathbf{k}_{\mathbf{r}}\right)\right. \\
& \left.+\left(k_{0} \frac{\mathbf{r}_{\mathbf{b}}}{\left|\mathbf{r}_{\mathbf{b}}\right|}+\mathbf{k}_{\mathbf{r}}\right)\right] \cdot \hat{\mathbf{n}}_{S} \times \frac{A(\mathbf{r})}{\hat{\mathbf{n}}_{S} \cdot \mathbf{z}} \mathrm{d}^{3} r e^{2 \pi j k_{0} \mathbf{r}^{\prime} \mid \mathbf{r}_{\mathbf{b}}} \frac{\mathrm{r}}{\mid \mathbf{r}_{\mathbf{b}}} \mathrm{d} s .
\end{aligned}
$$

Using the sifting properties of the Dirac delta function once again, the measured field can be written as the indefinite integral

$$
\begin{aligned}
E_{m}^{\prime}\left(\mathbf{r}^{\prime}\right)= & \frac{-k_{0}}{2} \int \frac{1}{\left|\mathbf{r}_{\mathbf{b}}\right|^{2}} \int e^{-2 \pi j\left(k_{0} \frac{\mathbf{r}_{\mathbf{b}}}{\mathbf{r}_{\mathbf{b}} \mid} \mathbf{k}_{\mathbf{r}}\right) \cdot \mathbf{r}}\left[R\left(k_{0} \frac{\mathbf{r}_{\mathbf{b}}}{\left|\mathbf{r}_{\mathbf{b}}\right|}-\mathbf{k}_{\mathbf{r}}\right)\right. \\
& \left.+\left(k_{0} \frac{\mathbf{r}_{\mathbf{b}}}{\left|\mathbf{r}_{\mathbf{b}}\right|}+\mathbf{k}_{\mathbf{r}}\right)\right] \cdot \hat{\mathbf{n}}_{S} \frac{A(\mathbf{r})}{\hat{\mathbf{n}}_{S} \cdot \mathbf{z}} \mathrm{d}^{3} r e^{2 \pi j k_{0} \mathbf{r}^{\prime} \frac{\mathbf{r}_{\mathbf{b}}}{\mid \mathrm{r}_{\mathbf{b}}}} \delta\left(\left|\mathbf{r}_{\mathbf{b}}\right|\right. \\
& \left.-r_{0}\right) \mathrm{d}^{3} r_{b} .
\end{aligned}
$$

Making the substitution, $\mathbf{k}^{\prime} / k_{0}=\mathbf{r}_{\mathbf{b}} / r_{0}$, it is found,

$$
\begin{aligned}
E_{m}^{\prime}\left(\mathbf{r}^{\prime}\right)= & -\frac{1}{2 k_{0}} \int\left[\int e^{-2 \pi j\left(\mathbf{k}^{\prime}-\mathbf{k}_{\mathbf{r}}\right) \cdot \mathbf{r}}\left[R\left(\mathbf{k}^{\prime}-\mathbf{k}_{\mathbf{r}}\right)+\left(\mathbf{k}^{\prime}+\mathbf{k}_{\mathbf{r}}\right)\right]\right. \\
& \left.\cdot \hat{\mathbf{n}}_{S} \frac{A(\mathbf{r})}{\hat{\mathbf{n}}_{S} \cdot \mathbf{z}} \mathrm{d}^{3} r\right] \delta\left(\left|\mathbf{k}^{\prime}\right|-k_{0}\right) e^{2 \pi j \mathbf{k}^{\prime} \cdot \mathbf{r}^{\prime}} \mathrm{d}^{3} k^{\prime}
\end{aligned}
$$

A further simplification can be made by considering the phase within the bracketed integral in Eq. (21). Since the phase of the complex exponential changes in the direction defined by $\left(\mathbf{k}^{\prime}-\mathbf{k}_{\mathbf{r}}\right)$, only regions of the surface where the surface normal is in this direction will contribute to the integral. This is the principle of stationary phase and is illustrated 


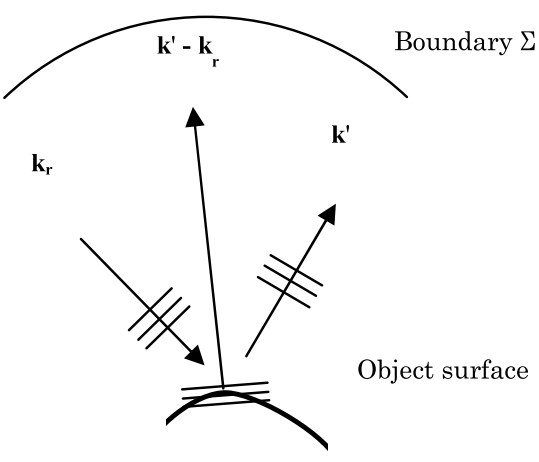

Fig. 3. Principle of stationary phase.

in Fig. 3. Noting that case in these regions the term $\left(\mathbf{k}^{\prime}+\mathbf{k}_{\mathbf{r}}\right) \cdot \hat{\mathbf{n}}_{S}$ is negligible and $\hat{\mathbf{n}}_{S}=\left(\mathbf{k}^{\prime}-\mathbf{k}_{r}\right) /\left|\mathbf{k}^{\prime}-\mathbf{k}_{\mathbf{r}}\right|$, Eq. (21) becomes

$$
\begin{aligned}
E_{m}^{\prime}\left(\mathbf{r}^{\prime}\right)= & -\frac{R}{2 k_{0}} \iint e^{-2 \pi j\left(\mathbf{k}^{\prime}-\mathbf{k}_{\mathbf{r}}\right) \cdot \mathbf{r}}\left(\frac{\left|\mathbf{k}^{\prime}-\mathbf{k}_{\mathbf{r}}\right|^{2}}{\left(\mathbf{k}^{\prime}-\mathbf{k}_{\mathbf{r}}\right) \cdot \mathbf{z}}\right) A(\mathbf{r}) \mathrm{d}^{3} r \delta\left(\left|\mathbf{k}^{\prime}\right|\right. \\
& \left.-k_{0}\right) e^{2 \pi j \mathbf{k}^{\prime} \cdot \mathbf{r}^{\prime}} \mathrm{d}^{3} k^{\prime} .
\end{aligned}
$$

With reference to Appendix $\underline{\mathrm{A}}$, an ideal imaging system that collects the field over the whole surface of the boundary sphere has a TF given by $\tilde{G}_{\text {ideal }}\left(\mathbf{k}^{\prime}\right)=$ $\left(j / 4 \pi k_{0}\right) \delta\left(\left|\mathbf{k}^{\prime}\right|-k_{0}\right)$. Making this substitution,

$$
\begin{aligned}
E_{m}^{\prime}\left(\mathbf{r}^{\prime}\right)= & 4 \pi j R \iint e^{-2 \pi j\left(\mathbf{k}^{\prime}-\mathbf{k}_{\mathbf{r}}\right) \cdot \mathbf{r}}\left(\frac{\left|\mathbf{k}^{\prime}-\mathbf{k}_{\mathbf{r}}\right|^{2}}{2\left(\mathbf{k}^{\prime}-\mathbf{k}_{\mathbf{r}}\right) \cdot \mathbf{z}}\right) A(\mathbf{r}) \mathrm{d}^{3} r \\
& \tilde{G}_{\text {ideal }}\left(\mathbf{k}^{\prime}\right) e^{2 \pi j \mathbf{k}^{\prime} \cdot \mathbf{r}^{\prime}} \mathrm{d}^{3} k^{\prime} .
\end{aligned}
$$

For an instrument of limited numerical aperture, however, the TF is $\tilde{G}_{\mathrm{NA}}\left(\mathbf{k}^{\prime}, k_{0}\right)=\left(j / 4 \pi k_{0}\right) \delta\left(\left|\mathbf{k}^{\prime}\right|-k_{0}\right)$ $\operatorname{step}\left(\left(\mathbf{k}^{\prime} \cdot \hat{\boldsymbol{o}} / k_{0}\right)-\sqrt{1-N_{A}^{2}}\right)$, and the measured field $E_{m}\left(\mathbf{r}^{\prime}\right)$ is given by

$$
\begin{aligned}
E_{m}\left(\mathbf{r}^{\prime}\right)= & 4 \pi j R \iint e^{-2 \pi j\left(\mathbf{k}^{\prime}-\mathbf{k}_{\mathbf{r}}\right) \cdot \mathbf{r}}\left(\frac{\left|\mathbf{k}^{\prime}-\mathbf{k}_{\mathbf{r}}\right|^{2}}{2\left(\mathbf{k}^{\prime}-\mathbf{k}_{\mathbf{r}}\right) \cdot \mathbf{z}}\right) A(\mathbf{r}) \mathrm{d}^{3} r \\
& \tilde{G}_{\mathrm{NA}}\left(\mathbf{k}^{\prime}, k_{0}\right) e^{2 \pi j \mathbf{k}^{\prime} \cdot \mathbf{r}^{\prime}} \mathrm{d}^{3} k^{\prime} .
\end{aligned}
$$

Equation (24) describes the field measured by an unspecified coherent instrument operating in the far-field with restricted numerical aperture, when the surface of interest is illuminated by a plane monochromatic wave propagating in the direction of the wave vector, $\mathbf{k}_{\mathbf{r}}$. In order to derive an expression for the response of a CSI, it is now necessary to consider this type of instrument in more detail. CSI records the interference between light scattered from the surface of interest and that reflected from a reference flat as the surface is scanned through focus (i.e., scanned in the axial direction). Typically, a Mirau objective utilizing an internal reference flat, as shown in Fig. 4, is used for this purpose.

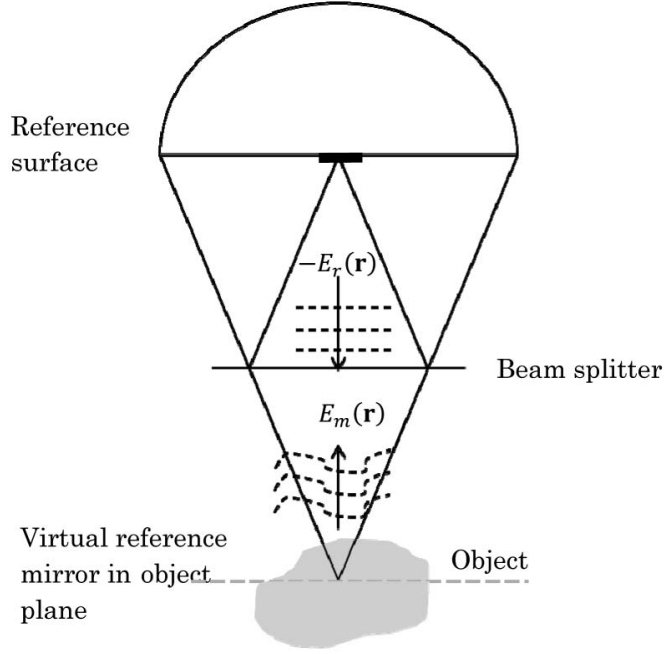

Fig. 4. Superposition of the reference and scattered fields in a Mirau objective.

In this way, the intensity recorded by the camera is proportional to that in the object plane of the objective, which is a far-field measurement of the interference between the measured scattered field $E_{m}(\mathbf{r})$ and the reference field $-E_{r}(\mathbf{r})$, as shown in Fig. 4. Note that the reference field is $-E_{r}(\mathbf{r})$ due to reflection at the reference surface. Accordingly, the measured intensity, $I(\mathbf{r})$, in the resulting interferogram is given by

$$
\begin{aligned}
I(\mathbf{r}) & =\left|E_{m}(\mathbf{r})-E_{r}(\mathbf{r})\right|^{2} \\
& =\left|E_{r}(\mathbf{r})\right|^{2}+\left|E_{m}(\mathbf{r})\right|^{2}-E_{m}(\mathbf{r})^{*} E_{r}(\mathbf{r})-E_{m}(\mathbf{r}) E_{r}(\mathbf{r})^{*} .
\end{aligned}
$$

In a similar manner to off-axis holography, these terms are separable in the frequency domain (see for example [17]) so the output of a CSI instrument is defined as the modulated (fringe) component of the interferogram given by the fourth term in Eq. (25),

$$
O(\mathbf{r})=E_{m}(\mathbf{r}) E_{r}(\mathbf{r})^{*} .
$$

Returning to the expression obtained for surface scattering with plane wave illumination, the output is, $O_{F}\left(\mathbf{r}^{\prime}\right)=E_{m}\left(\mathbf{r}^{\prime}\right) e^{-2 \pi j \mathbf{k}_{\mathbf{r}} \cdot \mathbf{r}^{\prime}}$ and substituting the measured scattered field $E_{m}\left(\mathbf{r}^{\prime}\right)$ from Eq. (24), then

$$
\begin{gathered}
O_{F}\left(\mathbf{r}^{\prime}\right)=\iint e^{-2 \pi j\left(\mathbf{k}^{\prime}-\mathbf{k}_{\mathbf{r}}\right) \cdot \mathbf{r}}\left(\frac{\left|\mathbf{k}^{\prime}-\mathbf{k}_{\mathbf{r}}\right|^{2}}{2\left(\mathbf{k}^{\prime}-\mathbf{k}_{\mathbf{r}}\right) \cdot \mathbf{z}}\right) \Delta_{F}(\mathbf{r}) \mathrm{d}^{3} r \\
\tilde{G}_{\mathrm{NA}}\left(\mathbf{k}^{\prime}, k_{0}\right) e^{2 \pi j\left(\mathbf{k}^{\prime}-\mathbf{k}_{\mathbf{r}}\right) \cdot \mathbf{r}^{\prime}} \mathrm{d}^{3} k^{\prime},
\end{gathered}
$$

where the surface is defined by the function, $\Delta_{F}(\mathbf{r})$, given by

$$
\Delta_{F}(\mathbf{r})=4 \pi j R A(\mathbf{r})=4 \pi j R W\left(r_{x}, r_{y}\right) \delta\left(r_{z}-s\left(r_{x}, r_{y}\right)\right) .
$$


Equation (28) provides a mathematical definition of the foil model of the surface. Making the substitution $\mathbf{k}^{\prime}-\mathbf{k}_{\mathbf{r}}=\mathbf{k}$ in Eq. (27) gives

$$
\begin{aligned}
O_{F}\left(\mathbf{r}^{\prime}\right)= & \iint \Delta_{F}(\mathbf{r}) e^{-2 \pi j \mathbf{k}^{\prime} \cdot \mathbf{r}} \mathrm{d}^{3} r\left(\frac{|\mathbf{k}|^{2}}{2 \mathbf{k} \cdot \mathbf{z}}\right) \tilde{G}_{\mathrm{NA}}(\mathbf{k} \\
& \left.+\mathbf{k}_{\mathbf{r}}, k_{0}\right) e^{2 \pi j \mathbf{k} \cdot \mathbf{r}^{\prime}} \mathrm{d}^{3} k
\end{aligned}
$$

or

$$
\tilde{O}_{F}(\mathbf{k})=\tilde{\Delta}_{F}(\mathbf{k})\left(\frac{|\mathbf{k}|^{2}}{2 \mathbf{k} \cdot \mathbf{z}}\right) \tilde{G}_{\mathrm{NA}}\left(\mathbf{k}+\mathbf{k}_{\mathbf{r}}, k_{0}\right) .
$$

Finally, if the spectral density of the source as a function of wavenumber is $S\left(k_{0}\right)$, then integrating over all illumination wave vectors, $\mathbf{k}_{\mathbf{r}}$, within the numerical aperture and all wavenumbers, $k_{0}$ that are defined by the function $\tilde{G}_{\mathrm{NA}}\left(-\mathbf{k}_{\mathbf{r}}, k_{0}\right)$, the output of the CSI can be written

$$
\tilde{O}_{F}(\mathbf{k})=\tilde{\Delta}_{F}(\mathbf{k}) \tilde{H}_{F}(\mathbf{k}),
$$

where $\tilde{H}(\mathbf{k})$ is the TF and is given by

$$
\begin{aligned}
\tilde{H}_{F}(\mathbf{k})= & \left(\frac{|\mathbf{k}|^{2}}{2 \mathbf{k} \cdot \mathbf{z}}\right) \iint \tilde{G}_{\mathrm{NA}}\left(\mathbf{k}_{\mathbf{r}}, k_{0}\right) \tilde{G}_{\mathrm{NA}}(\mathbf{k} \\
& \left.-\mathbf{k}_{\mathbf{r}}, k_{0}\right) \mathrm{d}^{3} k_{r} S\left(k_{0}\right) \mathrm{d} k_{0} .
\end{aligned}
$$

Equation (32) defines the response of a CSI of restricted numerical aperture when it is applied to the foil model of an object surface as defined by Eq. (28).

\section{Discussion}

The TF defined by Eq. (32) is entirely equivalent to the "effective transfer function" presented by Sheppard in the context of confocal microscopy $[24,25]$. It should be compared with the expression for the TF derived using the Born approximation, Eq. (5), which is repeated here for clarity:

$$
\tilde{H}_{B}(\mathbf{k})=\iint \tilde{G}_{\mathrm{NA}}\left(\mathbf{k}^{\prime}, k_{0}\right) \tilde{G}_{\mathrm{NA}}\left(\mathbf{k}-\mathbf{k}^{\prime}, k_{0}\right) \mathrm{d}^{3} k^{\prime} k_{0}^{2} S\left(k_{0}\right) \mathrm{d} k_{0} .
$$

Although the forms of Eqs. (32) and (33) are similar, they differ in the weighting term, $\left(|\overline{\mathbf{k}}|^{2} /(2 \mathbf{k} . \mathbf{z})\right)$ in Eq. (32) and the factor $k_{0}^{2}$ that weights the spectrum of the illumination source, $S\left(k_{0}\right)$, in Eq. (33). It should also be noted that in each derivation the object function to which the filtering operation is applied is defined in a slightly different way. In the case of the Born approximation

$$
\Delta_{B}(\mathbf{r})=4 \pi^{2}\left(1-n^{2}(\mathbf{r})\right),
$$

whereas the foil model of the object is defined by

$$
\Delta_{F}(\mathbf{r})=4 \pi j R W\left(r_{x}, r_{y}\right) \delta\left(r_{z}-s\left(r_{x}, r_{y}\right)\right) .
$$

It should be remembered that the foil model is strictly only valid for perfect conductors when the reflection coefficient is independent of incidence angle and $R=1$. However, for small instruments of low numerical aperture, the angle of incidence is restricted and it is reasonable to replace the reflection coefficient with its value at normal incidence, $R=(1-n) /(1+n)$, such that

$$
\Delta_{F}(\mathbf{r}) \approx 4 \pi j\left(\frac{1-n}{1+n}\right) \delta\left(r_{z}-s\left(r_{x}, r_{y}\right)\right) .
$$

Finally, returning to Eq. (27) it is noted that for low numerical aperture, and consequently small angles of incidence, the factor $\left|\mathbf{k}^{\prime}-\mathbf{k}_{\mathbf{r}}\right|^{2} / 2\left(\mathbf{k}^{\prime}-\mathbf{k}_{\mathbf{r}}\right) \cdot \mathbf{z} \approx k_{0}$, and following a similar derivation

$\tilde{H}_{F}(\mathbf{k}) \approx \iint \tilde{G}_{\mathrm{NA}}\left(\mathbf{k}_{\mathbf{r}}, k_{0}\right) \tilde{G}_{\mathrm{NA}}\left(\mathbf{k}-\mathbf{k}_{\mathbf{r}}, k_{0}\right) \mathrm{d}^{3} k_{r} k_{0} S\left(k_{0}\right) \mathrm{d} k_{0}$.

Equivalently, the PSF is given by

$$
H_{F}(\mathbf{r}) \approx \int G_{\mathrm{NA}}^{2}\left(\mathbf{r}, k_{0}\right) k_{0} S\left(k_{0}\right) \mathrm{d} k_{0} .
$$

Equations (36)-(38) define the properties of the foil model of the surface that is used in practice and it is straightforward to show that in the limit $n \rightarrow 1$ and $N_{A} \rightarrow 0$ the output interferograms of a CSI that are predicted using the Born approximation and foil model are in fact identical.

\section{Conclusion}

In this paper, the process of surface measurement using CSI in terms of linear systems theory has been discussed. The interferogram that is output by a CSI instrument is defined as a linear filtering operation applied to an appropriate object function that is defined in terms of the geometry and optical properties of the object. The filter is characterized by the PSF in the space domain or equivalently the $\mathrm{TF}$ in the frequency domain. As has been shown elsewhere $[17,18]$, these characteristics can be deduced for a range of far-field measurement instruments, including CSI, if the scattered field is a linear function of the object function, but in general, this is only true if some approximations are made. In this respect it is usual to derive the 3D PSF and TF by assuming the Born approximation. This is appropriate when the object is weakly scattering such that small changes in refractive index are observed but is not applicable to most $3 \mathrm{D}$ objects that scatter more strongly. For the case of surface scattering it is not necessary to make the Born approximation, however, and the process is found to be linear if appropriate boundary conditions 
are assumed. In this case the Kirchhoff or physical optics approximation is assumed, which implies that the surface is slowly varying (such that the local radius of curvature is larger than the wavelength). When the surface of a homogenous object is measured, it can be replaced by an infinitely thin foil-like membrane, which has been called the "foil model" of the surface.

The paper presents for the first time the derivation of the foil model of the surface and the associated PSF and TF. Starting from the integral form of the Helmholtz equation the differences between the approaches based on the Born and Kirchhoff approximations are described. It is shown that there is a small but significant difference between the PSFs and TFs; however, the two approaches yield exactly the same result when both the numerical aperture and refractive change tends to zero.

\section{Appendix A}

In the following it is shown that the reconstruction of a monochromatic scattered field from the information present within a measurement of the complex amplitude at a distant boundary surface can be expressed as a linear filtering operation. The transfer characteristic of this operation is derived both for the ideal case of complete recording of the boundary field and an incomplete recording over a surface restricted by numerical aperture.

Consider the field, $E_{s}\left(\mathbf{r}_{\mathbf{b}}\right)$, at a spherical boundary due to an object described by the source distribution, $U(\mathbf{r})$, as shown in Fig. 5. According to scalar scattering theory this can be written [17] as the superposition integral

$$
E_{s}\left(\mathbf{r}_{\mathbf{b}}\right)=\int U(\mathbf{r}) G\left(\mathbf{r}_{\mathbf{b}}-\mathbf{r}\right) \mathrm{d}^{3} r,
$$

where $G\left(\mathbf{r}_{\mathbf{b}}-\mathbf{r}\right)$ is the free-space Green's function that defines a point source and is given by

$$
G\left(\mathbf{r}_{\mathbf{b}}-\mathbf{r}\right)=\frac{e^{2 \pi j k_{0}\left|\mathbf{r}_{\mathbf{b}}-\mathbf{r}\right|}}{4 \pi\left|\mathbf{r}_{\mathbf{b}}-\mathbf{r}\right|},
$$

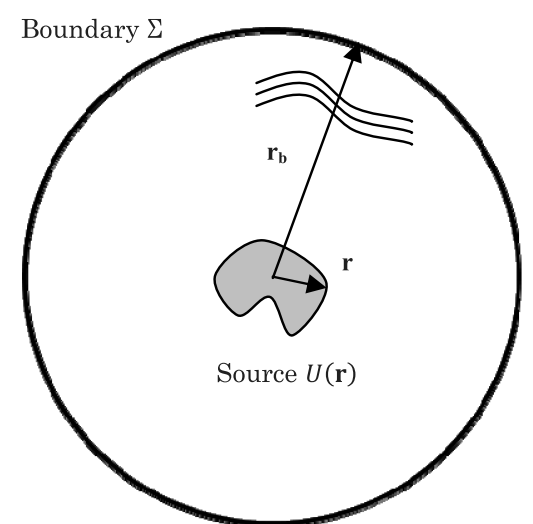

Fig. 5. Scattered field at spherical boundary surface. and $k_{0}=1 / \lambda$ is the wavenumber. If the boundary of interest is placed in the far field, such that $\left|\mathbf{r}_{\mathbf{b}}\right| \gg|\mathbf{r}|$, the free-space Green's function can be written

$$
G\left(\mathbf{r}_{\mathbf{b}}-\mathbf{r}\right) \approx \frac{e^{2 \pi j k_{0}\left|\mathbf{r}_{\mathbf{b}}\right|}}{4 \pi\left|\mathbf{r}_{\mathbf{b}}\right|} e^{-2 \pi j k_{0} \cdot \mathbf{r} \cdot \frac{\mathbf{r}_{\mathbf{b}}}{\mathrm{r}_{\mathbf{b}}}},
$$

where it is noted that this approximation is equivalent to the Fraunhofer approximation [18]. Using Eq. (A3) the scattered field is given by

$$
E_{s}\left(\mathbf{r}_{\mathbf{b}}\right)=\frac{e^{2 \pi j k_{0}\left|\mathbf{r}_{\mathbf{b}}\right|}}{4 \pi\left|\mathbf{r}_{\mathbf{b}}\right|} \int U(\mathbf{r}) e^{-2 \pi j k_{0} \mathbf{r} \cdot \frac{\mathbf{r}_{\mathbf{b}}}{\cdot \mathbf{r}_{\mathbf{b}}}} \mathrm{d}^{3} r
$$

Consider the reconstruction of the scattered field using the information contained within a measurement of the complex amplitude on the boundary surface. This could be an optical reconstruction corresponding to the real image reconstructed from a holographic recording medium placed around the boundary, or equivalently, a numerical reconstruction from a digital recording of the boundary field. In either case, the reconstructed field is found by considering the back-propagation of the boundary field. Accordingly, a point sink defined by the conjugate of the Green's function in Eq. (A33) is applied to the Kirchhoff integral [19],

$E_{m}\left(\mathbf{r}^{\prime}\right)=\int_{\Sigma}\left[G^{*}\left(\mathbf{r}^{\prime}-\mathbf{r}_{\mathbf{b}}\right) \frac{\partial E_{S}\left(\mathbf{r}_{\mathbf{b}}\right)}{\partial n}-E_{S}\left(\mathbf{r}_{\mathbf{b}}\right) \frac{\partial G^{*}\left(\mathbf{r}^{\prime}-\mathbf{r}_{\mathbf{b}}\right)}{\partial n}\right] \mathrm{d} s$,

where $\partial / \partial n$ is the field derivative in the direction of the outward surface normal and the surface integral is performed over the boundary surface $\Sigma$. Without loss of generality, assuming the boundary is spherical of radius $\mathbf{r}_{0}$, and using the far-field approximation once again gives

$$
\frac{\partial G^{*}\left(\mathbf{r}^{\prime}-\mathbf{r}_{\mathbf{b}}\right)}{\partial n}=-2 \pi j k_{0} G^{*}\left(\mathbf{r}^{\prime}-\mathbf{r}_{\mathbf{b}}\right),
$$

and

$$
\frac{\partial E_{S}\left(\mathbf{r}_{\mathbf{b}}\right)}{\partial n}=2 \pi j k_{0} E_{S}\left(\mathbf{r}_{\mathbf{b}}\right) .
$$

Substituting values from Eqs. (A6) and (A7) to Eq. (A5) the reconstructed field is given by

$$
\begin{aligned}
E_{m}\left(\mathbf{r}^{\prime}\right) & =4 \pi j k_{0} \int_{\Sigma} G^{*}\left(\mathbf{r}^{\prime}-\mathbf{r}_{\mathbf{b}}\right) E_{S}\left(\mathbf{r}_{\mathbf{b}}\right) \mathrm{d} s \\
& =\frac{j k_{0}}{4 \pi} \int_{\Sigma}\left[\int U(\mathbf{r}) e^{-2 \pi j k_{0} \cdot \mathbf{r} \cdot \frac{\mathbf{r}_{\mathbf{b}}}{\mathbf{r}_{\mathbf{b}}}} \mathrm{d}^{3} r\right] \frac{1}{\left|\mathbf{r}_{\mathbf{b}}\right|^{2}} e^{2 \pi j k_{0} \mathbf{r}^{\prime} \cdot \frac{\mathbf{r}_{\mathbf{b}}}{\mathbf{r}_{\mathbf{b}}}} \mathrm{d} s .
\end{aligned}
$$

Using the sifting properties of the Dirac Delta function [25], Eq. (A8) can be written as the indefinite integral 


$$
\begin{aligned}
E_{m}\left(\mathbf{r}^{\prime}\right)= & \frac{j k_{0}}{4 \pi} \int\left[\int U(\mathbf{r}) e^{-2 \pi j k_{0} \mathbf{r} \cdot \frac{\mathbf{r}_{\mathbf{b}}}{\mathbf{r}_{\mathbf{b}}}} \mathrm{d}^{3} r\right] \frac{1}{\left|\mathbf{r}_{\mathbf{b}}\right|^{2}} \delta\left(\left|\mathbf{r}_{\mathbf{b}}\right|\right. \\
& \left.-r_{0}\right) e^{2 \pi j k_{0} \mathbf{r}^{\prime} \cdot \frac{\mathbf{r}_{\mathbf{b}}}{r_{\mathbf{b}}} \mid} \mathrm{d}^{3} r_{b} .
\end{aligned}
$$

Finally, making the substitution, $\mathbf{k}^{\prime} / k_{0}=\mathbf{r}_{\mathbf{b}} / r_{0}$, it is found that

$$
\begin{aligned}
E_{m}\left(\mathbf{r}^{\prime}\right)= & \frac{j}{4 \pi k_{0}} \int\left[\int U(\mathbf{r}) e^{-2 \pi j \mathbf{k} \cdot \mathbf{r}} \mathrm{d}^{3} r\right] \delta(|\mathbf{k}| \\
& \left.-k_{0}\right) e^{2 \pi j \mathbf{k} \cdot \mathbf{r}^{\prime}} \mathrm{d}^{3} k .
\end{aligned}
$$

In this expression the bracketed term can be recognized as the source spectrum $\tilde{U}(\mathbf{k})$. Taking the Fourier transform of Eq. (A10), the spectrum of the reconstructed field $\tilde{E}_{m}(\mathbf{k})$, can be written

$$
\tilde{E}_{m}(\mathbf{k})=\tilde{U}(\mathbf{k}) \tilde{G}_{\text {ideal }}(\mathbf{k}),
$$

where $\tilde{G}_{\text {ideal }}(\mathbf{k})$ is the TF given by

$$
\tilde{G}_{\text {ideal }}(\mathbf{k})=\frac{j}{4 \pi k_{0}} \delta\left(|\mathbf{k}|-k_{0}\right) .
$$

Consequently, the reconstruction process can be considered as a linear filtering operation that modifies the source spectrum by selecting only the spatial frequency components that lie on the sphere of radius $k_{0}$. In practice, however, most optical instruments collect light over a finite part of the sphere defined by the numerical aperture $N_{A}$. In this case the TF, $\tilde{G}_{\mathrm{NA}}\left(\mathbf{k}, k_{0}\right)$, can be represented by a portion of the sphere such that

$$
\tilde{G}_{\mathrm{NA}}\left(\mathbf{k}, k_{0}\right)=\frac{j}{4 \pi k_{0}} \delta\left(|\mathbf{k}|-k_{0}\right) \operatorname{step}\left(\frac{\mathbf{k} \cdot \hat{\boldsymbol{o}}}{k_{0}}-\sqrt{1-N_{A}^{2}}\right),
$$

where $\operatorname{step}(x)$ is the Heaviside step function and $\hat{\boldsymbol{o}}$ is a unit vector in the direction of observation (i.e., along the optical axis of the instrument). Equation (A13) defines the 3D TF of a far-field imaging system of finite aperture. The 3D PSF is the inverse Fourier transform of this expression and is given by

$$
\begin{aligned}
G_{\mathrm{NA}}\left(\mathbf{r}, k_{0}\right)= & \int \frac{j}{4 \pi k_{0}} \delta\left(|\mathbf{k}|-k_{0}\right) \operatorname{step}\left(\frac{\mathbf{k} \cdot \hat{\boldsymbol{o}}}{k_{0}}\right. \\
& \left.-\sqrt{1-N_{A}^{2}}\right) e^{j 2 \pi \mathbf{k} \cdot r} \mathrm{~d}^{3} k .
\end{aligned}
$$

Equations (A13) and (A14) define the response of a far-field imaging system of restricted numerical aperture as a 3D filter applied to the source distribution $U(\mathbf{r})$.

The authors wish to acknowledge the EPSRC and NPL for supporting this work through the BIS National Measurement System Engineering \& Flow Metrology Programme. Thanks are due to Dr.
Andrew Henning (NPL) for checking through the mathematics in detail.

\section{References}

1. B. S. Lee and T. C. Strand, "Profilometry with a coherence scanning microscope," Appl. Opt. 29, 3784-3788 (1990).

2. L. Deck and P. De Groot, "High-speed noncontact profiler based on scanning white-light interferometry," Appl. Opt. 33, 7334-7338 (1994).

3. R. K. Leach, Optical Measurement of Surface Topography (Springer, 2011), 187-208.

4. G. S. Kino and S. S. Chim, "Mirau correlation microscope," Appl. Opt. 29, 3775-3783 (1990).

5. F. Gao, R. K. Leach, J. Petzing, and J. M. Coupland, "Surface measurement errors using commercial scanning white light interferometers," Meas. Sci. Technol. 19, 015303 (2008).

6. C. L. Giusca and R. K. Leach, "Calibration of the scales of areal surface topography measuring instruments: Part 2-Amplification coefficient, linearity and squareness," Meas. Sci. Technol. 23, 065005 (2012).

7. J. N. Petzing, J. M. Coupland, and R. K. Leach, "The measurement of rough surface topography using coherence scanning interferometry," in Good Practice Guide No. 116, (National Physical Laboratory, 2010), pp. 92-112.

8. W. Hillmann, "Surface profiles obtained by means of optical methods-are they true representations of the real surface," CIRP Ann. 39, 581-583 (1990).

9. K. Palodhi, J. M. Coupland, and R. K. Leach, "A linear model of fringe generation and analysis in coherence scanning interferometry," presented at the ASPE Summer Topical Meeting on Precision Interferometric Metrology, Asheville, North Carolina, 23-25 June 2010.

10. K. Palodhi, J. M. Coupland, and R. K. Leach, "Determination of the point spread function of a coherence scanning interferometer," in Proceedings of the 13th International Conference on Metrology and Properties of Engineering Surfaces (NPL, 2011), pp. 199-202.

11. R. Mandal, K. Palodhi, J. M. Coupland, R. K. Leach, and D. Mansfield, "Application of linear systems theory to characterize coherence scanning interferometry," Proc. SPIE 8430, $84300 \mathrm{~T}$ (2012).

12. E. Wolf, "Three-dimensional structure determination of semitransparent objects from holographic data," Opt. Commun. 1, 153-156 (1969).

13. R. Dandliker and K. Weiss, "Reconstruction of the threedimensional refractive index from scattered waves," Opt. Commun. 1, 323-328 (1970).

14. N. Streibl, "Three-dimensional imaging by a microscope," J. Opt. Soc. Am. A 2, 121-127 (1985).

15. C. J. R. Sheppard and C. J. Cogswell, "Three-dimensional image formation in confocal microscopy," J. Microsc. 159, 179-194 (1990).

16. A. F. Fercher, H. Bartelt, H. Becker, and E. Wiltschko, "Image formation by inversion of scattered field data: experiments and computational simulation," Appl. Opt. 18, 2427-2439 (1979).

17. J. M. Coupland and J. Lobera, "Holography, tomography and 3D microscopy as linear filtering operations," Meas. Sci. Technol. 19, 07010 (2008).

18. P. D. Ruiz, J. M. Huntley, and J. M. Coupland, "Depth-resolved imaging and displacement measurement techniques viewed as linear filtering operations," Exp. Mech. 13, 453-465 (2010).

19. M. Born and E. Wolf, Principles of Optics: Electromagnetic Theory of Propagation, Interference and Diffraction of Light, 7th ed. (Cambridge University, 1999), 695-734.

20. P. Beckmann and A. Spizzichino, The Scattering of Electromagnetic Waves from Rough Surfaces (Artech House, 1987), $17-33$.

21. R. J. Wombellan and J. A. DeSanto, "Reconstruction of rough-surface profiles with the Kirchhoff approximation," J. Opt. Soc. Am. A 8, 1892-1897 (1991).

22. E. I. Thorsosand and D. R. Jackson, "Studies of scattering theory using numerical methods," Waves Random Media 1 , S165-S190 (1991). 
23. C. J. Raymond, "Milestones and future directions in applications of optical scatterometry," Proc. SPIE CR72, 147-177 (1999).

24. C. J. R. Sheppard and M. Gu, "The significance of 3-D transfer functions in confocal scanning microscopy," J. Microsc. 165, 377-390 (1992).
25. C. J. R. Sheppard, "Imaging of random surfaces and inverse scattering in the Kirchhoff approximation, waves in random media," Waves Random Media 8, 53-66 (1998).

26. J. D. Gaskill, Linear Systems, Fourier Transforms and Optics (Wiley, 1978), p. 56. 